\title{
Association between Anxiety and Functional Outcomes in Patients with Stroke: A 1-Year Longitudinal Study
}

\author{
Eun-Hye Lee ${ }^{1}$, Ju-Wan Kim${ }^{1}$, Hee-Ju Kang ${ }^{1}$, Sung-Wan Kim¹, Joon-Tae Kim², \\ Man-Seok Park ${ }^{2}$, Ki-Hyun $\mathrm{Cho}^{2}$, and Jae-Min Kim ${ }^{1}{ }^{凶}$ \\ 1'Department of Psychiatry, Chonnam National University Medical School, Gwangju, Republic of Korea \\ ${ }^{2}$ Department of Neurology, Chonnam National University Medical School, Gwangju, Republic of Korea
}

Objective Anxiety is one of the most common complications in patients with stroke, but studies on its relationship to functional outcomes are limited and controversial. We investigated the association between post-stroke anxiety (PSA) and a 1-year trajectory of functional outcome.

Methods A total of 423 patients were recruited within 2 weeks after a stroke (acute phase) during hospitalization. Of them, 306 (72.3\%) completed follow-up examinations 1 year thereafter (chronic phase). Anxiety was evaluated using the Hospital Anxiety and Depression Scale-Anxiety subscale, and functional outcomes were measured using the National Institutes of Health Stroke Scale for stroke severity, the Barthel Index for activities of daily living (ADL), and the Mini-Mental State Examination for cognitive function at 2 weeks and 1 year. A range of demographic and clinical covariates were considered. The cross-sectional and longitudinal associations between PSA and functional outcomes were investigated.

Results PSA at the acute phase was not associated with functional outcomes at the cross-sectional point, but predicted worsening of outcome on stroke severity and ADL 1 year after stroke. PSA at the chronic phase was cross-sectionally associated with poor functional outcomes in all three measures. All associations were independent of potential covariates.

Conclusion Screening for anxiety is recommended even during the acute phase of stroke, considering its independent detrimental effect on functional prognosis. Screening for anxiety during the chronic phase is also encouraged, as this may reflect the functional status of stroke.

Psychiatry Investig 2019;16(12):919-925

Key Words Stroke, Anxiety, Longitudinal study, Functional outcome.

\section{INTRODUCTION}

Stroke is a major cause of neurological disability, resulting in various negative psychological consequences, including depression, fatigue, and anxiety. Extensive research has been conducted on post-stroke depression (PSD), and patients with PSD generally show higher morbidity and mortality rates than those without PSD. ${ }^{1,2}$ Post-stroke anxiety (PSA) is also a significant psychiatric symptom in stroke patients, affecting more than one-quarter of stroke survivors. ${ }^{3}$ However,

Received: July 26, 2019 Revised: August 27, 2019

Accepted: October 1, 2019

$\triangle$ Correspondence: Jae-Min Kim, MD, PhD

Department of Psychiatry, Chonnam National University Medical School, 42 Jebong-ro, Dong-gu, Gwangju 61469, Republic of Korea

Tel: +82-62-220-6143, Fax: +82-62-225-2351, E-mail: jmkim@chonnam.ac.kr

(c) This is an Open Access article distributed under the terms of the Creative Commons Attribution Non-Commercial License (https://creativecommons.org/licenses/bync/4.0) which permits unrestricted non-commercial use, distribution, and reproduction in any medium, provided the original work is properly cited. in contrast to PSD, studies on PSA and its relationship to functional outcomes, including stroke severity, activities of daily living (ADLs), and cognitive function are relatively limited, and have drawn inconsistent conclusions.

An early study reported that stroke patients with generalized anxiety had no significant differences in social, cognitive, or physical functions, compared to the those without anxiety after more than 2 years of follow-up after stroke. ${ }^{4}$ However, in the same dataset, PSA was significantly related to the impairment of ADLs during the acute phase but not the chronic phase of stroke. ${ }^{5}$ Two studies reported no association between anxiety measured within 6 months after stroke and functional recovery 5-10 years later. ${ }^{6,7}$ However, positive findings have been reported in more recent studies. One study reported that anxiety during the acute phase of stroke was significantly associated with lower levels of ADLs 3 months after stroke. ${ }^{8}$ Another study reported that PSA significantly predicted poor functional outcomes even 10 years after stroke. ${ }^{9}$ However, 
these studies assessed functional outcomes only by the level of ADLs or used the modified Rankin scale.

Previous studies were limited in terms of heterogeneous evaluation points, not being comprehensive investigations on functional outcomes, and using just one anxiety estimate, all of which may have rendered inconsistent findings. ${ }^{4-9}$ Therefore, the present study investigated the impact of PSA on three major categories of functional outcomes, cognitive function, physical ADLs, and neurological impairment, during the acute ( 2 weeks) and chronic phases (1 year) after the index stroke.

\section{METHODS}

\section{Study outline}

This analysis is part of a larger parent study designed to investigate psychiatric disorders of stroke patients hospitalized in the Department of Neurology at Chonnam National University Hospital, Gwangju, South Korea. Participants were individually evaluated 2 weeks and 1 year post-stroke. Anxiety and functional outcomes were evaluated 2 weeks and 1 year after stroke. Demographic and clinical characteristics were collected at 2 weeks.

\section{Participants}

Participants were consecutively recruited from all patients with recent ischemic stroke hospitalized within the Department of Neurology at Chonnam National University Hospital, Gwangju, South Korea between March 2006 and December 2009. The eligible participants were recruited about 2 weeks after the initial diagnosis of stroke. The inclusion criteria were: 1) patients with an ischemic stroke confirmed by a neuroimaging technique, such as brain magnetic resonance imaging (MRI) and computed tomography (CT), within 7 days; 2) patients that could adequately cooperate with staff and complete the questionnaires and assessments; and 3) patients capable of fully comprehending the objective of this study and providing informed consent. The exclusion criteria were: 1) patients with a severe physical illness that might lead to a life-threatening condition or patients whose course of recovery might be interrupted by this study; 2) patients with communication difficulties due to dysphagia or severe hearing impairment; 3) patients with any of the following comorbid neuropsychiatric conditions: dementia, Parkinson's disease, psychosis, substance abuse, brain tumor, or epilepsy; 4) patients with a pre-existing physical restriction prior to stroke; and 5) patients with a Mini Mental State Examination (MMSE) score $<16$. This study strictly followed the code of ethics enforced by the Institutional Review Board of Chonnam National University Hospital (IRB No. I-2008-02-028), and all participants provided written informed consent.

\section{Measurement and assessment of anxiety}

The Hospital Anxiety and Depression Scale (HADS) ${ }^{10}$ was used to determine anxiety symptoms. The HADS was created to assess patients with a physical comorbidity using two 7 -item subscales that evaluate depression and anxiety. Individual items are rated on a 4 -point $(0-3)$ scale. The total score (HADS-T; 0-42) reflects scores on two subscales: a score of 0-21 for the anxiety subscale (HADS-A) and a score of 0-21 for the depression subscale (HADS-D). HADS-A was employed to assess the levels of anxiety in the participants. The presence of PSA was defined as a HADS-A score $\geq 7$, as described previously. ${ }^{7,11,12}$ HADS has been validated in stroke patients and the optimal cut-off has been presented as HADSA score $\geq 7 . .^{11,12}$ The HADS has been formally translated and standardized in Korean. ${ }^{13}$ Each individual participant completed the HADS-A 2 weeks and 1 year after their stroke to evaluate the acute and chronic states of anxiety, respectively.

\section{Measurement and assessment of functional outcome post-stroke}

All participants were evaluated for functional outcomes in three categories: neurological severity, physical disability, and cognitive impairment.

\section{National Institutes of Health Stroke Scale (NIHSS)}

The NIHSS ${ }^{14}$ is a systematic assessment tool that provides a quantitative measure of stroke-related neurological deficits. The NIHSS is composed of 11 items that measure motor and sensory functions, language and speech production, vision, level of consciousness, and attention and neglect. Each item assesses a specific functional ability based on a score from 0 to 4 . For each item, a lower score indicates normal, whereas a higher score indicates a higher level of neurological severity.

\section{Barthel Index (BI)}

The $\mathrm{BI}^{15}$ is an objective tool based on an ordinal scale to measure performance of ADLs. The BI is composed of 10 variables that indicate the following ADLs: mobility, feeding, toilet use, dressing, bathing, fecal and urinary incontinence, help with grooming, and other activities. A higher number is associated with a greater likelihood of achieving independence with ADLs after discharge. Each item is scored as 0, 5, or 10 points; therefore, BI scores can range from 0 to 100 , corresponding to five levels of physical restrictions in ADLs: independence (100 points), low dependence (91-99 points), moderate dependence (61-90 points), severe dependence (21-60 points), and total dependence (0-20).

\section{Mini Mental Status Examination (MMSE)}

The $\mathrm{MMSE}^{16}$ measures cognitive function and is composed 
of 30 questions. A score of 1 indicates a positive outcome whereas a score of 0 indicates a negative outcome for each item. The MMSE measures the following domains of functional abilities: registration, attention and calculation, recall, language, ability to follow simple commands, and orientation. Lower scores represent greater cognitive impairment.

\section{Demographic and clinical characteristics}

The following demographic and clinical characteristics associated with PSA ${ }^{7,17-19}$ were recorded: age, gender, years of education, history of depression and stroke, stroke localization by hemisphere (left, right, or bilateral), and location (anterior, posterior, or both) according to neuroimaging, such as brain MRI or CT. Vascular risk or disease was evaluated as hypertension, diabetes, heart disease, and hypercholesterolemia. Body mass index and smoking status were recorded. Depression was assessed by a structured psychiatric interview according to the Diagnostic and Statistical Manual of Mental Disorders, Fourth Edition criteria using the Mini International Neuropsychiatric Interview ${ }^{20}$ and depressive symptoms were assessed by the Hamilton Depression Rating Scale (HAMD). ${ }^{21}$

\section{Statistical analysis}

The dependent variables were PSA 2 weeks and 1 year after stroke. The demographic and clinical characteristics at baseline were compared by PSA status using the t-test or the chisquared $\left(\chi^{2}\right)$ test, as appropriate, at each evaluation point. All variables significantly associated $(\mathrm{p}<0.05)$ with PSA at each point were considered covariates in later adjusted analyses. Cross-sectional associations between PSA and functional outcomes during the acute and chronic phases of stroke were analyzed using a t-test in the unadjusted model, and by logistic regression tests after adjusting for potential covariates. Finally, the longitudinal associations between acute phase PSA and changes in functional outcomes over 1 year were assessed using repeated-measures analysis of variance (ANOVA) in the same adjusted model. All statistical analyses were carried out using SPSS 23.0 software (IBM Corp., Armonk, NY, USA).

\section{RESULTS}

\section{Recruitment}

The recruitment procedure of the present study is shown in Figure 1. Of the 465 consecutively enrolled stroke patients who met the inclusion and exclusion criteria, 423 (91\%) consented to participate in the study. No significant differences in demographic or clinical characteristics were found between the participants and nonparticipants at this stage (all $\mathrm{p}>0.1$ ). A total of 306 of 423 participants $(72.3 \%)$ successfully

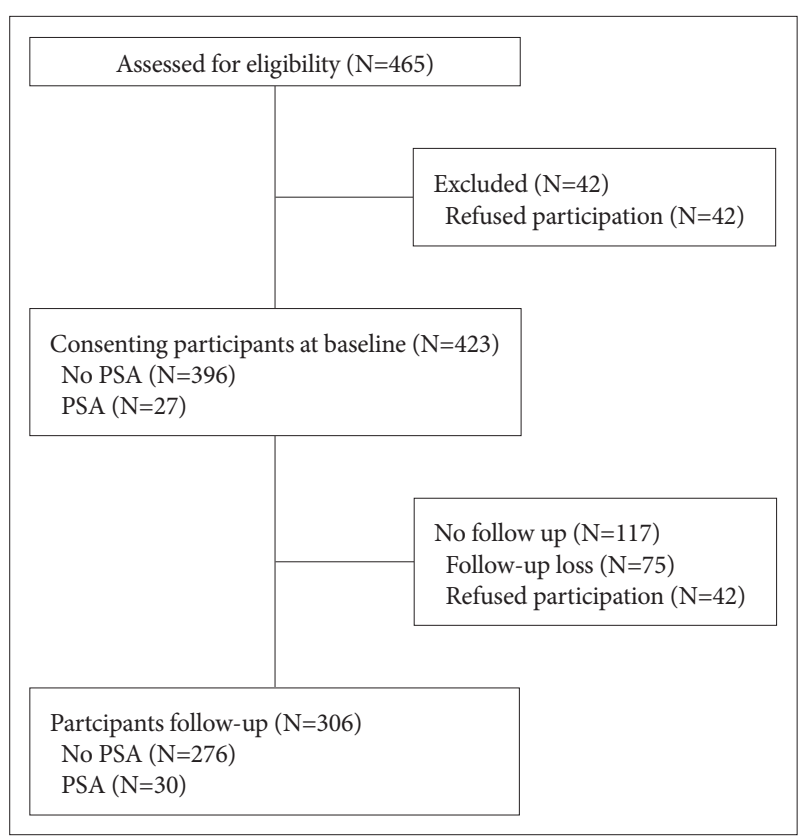

Figure 1. Study outline and recruitment process. PSA: PostStroke Anxiety.

completed the follow-up assessment at 1 year. The baseline demographic and clinical characteristics were not significantly different between those who were and were not available for the follow up assessment (all $\mathrm{p}>0.1$ ).

\section{Demographic and clinical characteristics by PSA}

PSA was diagnosed in $27(6.4 \%)$ of 423 and $30(9.8 \%)$ of 306 participants 2 weeks and 1 year after the index stroke, respectively. The baseline demographic and clinical characteristics were compared by PSA status at each evaluation point in Table 1. The presence of PSA at the acute phase was significantly associated with female gender, history of depression, and a higher HAMD score. The presence of PSA at the chronic phase was significantly associated with a higher HAMD score at baseline.

\section{Cross-sectional associations between PSA and functional outcomes}

Cross-sectional associations between PSA and functional outcomes during both the acute and chronic phases of stroke are summarized in Table 2. PSA at the acute phase was significantly associated with a lower MMSE score at baseline in the unadjusted analysis. However, the strength of the association lost significance after adjusting for gender, history of depression, and the HAMD score. PSA during the chronic phase was significantly associated with a higher NIHSS score, and lower BI and MMSE scores at follow-up in both unadjusted and adjusted analyses using the same model. 
Table 1. Baseline characteristics according to anxiety 2 weeks and at 1 year after stroke

\begin{tabular}{|c|c|c|c|c|c|c|}
\hline & \multicolumn{3}{|c|}{ Acute (2 weeks) } & \multicolumn{3}{|c|}{ Chronic (12 months) } \\
\hline & No PSA $(\mathrm{N}=396)$ & PSA $(\mathrm{N}=27)$ & p-value* & No PSA $(\mathrm{N}=276)$ & PSA $(\mathrm{N}=30)$ & p-value* \\
\hline Age, mean (SD) years & $64.6(10.1)$ & $62.4(9.3)$ & 0.237 & $63.6(9.8)$ & $64.3(10.9)$ & 0.746 \\
\hline Gender, N (\%) female & $159(40.2)$ & $20(74.1)$ & 0.001 & $109(39.5)$ & $17(56.7)$ & 0.069 \\
\hline Education, mean (SD) year & $8.6(4.9)$ & $7.1(4.6)$ & 0.113 & $8.8(4.9)$ & $7.4(5.2)$ & 0.176 \\
\hline Previous depression, N (\%) & $13(3.3)$ & $4(14.8)$ & 0.003 & $9(3.3)$ & $3(10.0)$ & 0.071 \\
\hline Previous stroke, N (\%) & $31(7.8)$ & $4(14.8)$ & 0.202 & $20(7.2)$ & $5(16.7)$ & 0.074 \\
\hline \multicolumn{7}{|l|}{ Stroke hemisphere, N (\%) } \\
\hline Left & $178(44.9)$ & $15(55.6)$ & 0.185 & $134(48.6)$ & $13(43.3)$ & 0.848 \\
\hline Right & $201(50.8)$ & $9(33.3)$ & & $132(47.8)$ & $16(53.3)$ & \\
\hline Bilateral & $17(4.3)$ & $3(11.1)$ & & $10(3.6)$ & $1(3.3)$ & \\
\hline \multicolumn{7}{|l|}{ Stroke location, $\mathrm{N}(\%)$} \\
\hline Anterior & $216(54.5)$ & $18(66.7)$ & 0.126 & $152(55.1)$ & $21(70.0)$ & 0.272 \\
\hline Posterior & $146(36.9)$ & $5(18.5)$ & & $103(37.3)$ & $8(26.7)$ & \\
\hline Both & $34(8.6)$ & $4(14.8)$ & & $21(7.6)$ & $1(3.3)$ & \\
\hline \multicolumn{7}{|l|}{ Vascular risk/disease, $\mathrm{N}(\%)$} \\
\hline Hypertension & $194(49.0)$ & $13(48.1)$ & 0.933 & $134(48.6)$ & $17(56.7)$ & 0.398 \\
\hline Diabetes & $110(27.8)$ & $6(22.2)$ & 0.531 & $76(27.5)$ & $8(26.7)$ & 0.919 \\
\hline Heart disease & $33(8.3)$ & $3(11.1)$ & 0.617 & $26(9.4)$ & $2(6.7)$ & 0.619 \\
\hline Hypercholesterolemia & $221(55.8)$ & $15(55.6)$ & 0.980 & $164(59.4)$ & $15(50.0)$ & 0.320 \\
\hline Body mass index, mean (SD) kg/m² & $24.1(2.8)$ & $24.3(2.9)$ & 0.664 & $24.3(2.8)$ & $23.8(3.0)$ & 0.366 \\
\hline Smoking, N (\%) current smoker & $66(16.7)$ & $3(11.1)$ & 0.127 & $52(18.8)$ & $4(13.3)$ & 0.706 \\
\hline Hamilton Depression Rating Scale at 2 weeks & $6.5(5.2)$ & $18.0(5.8)$ & $<0.001$ & $6.6(5.3)$ & $11.6(6.7)$ & $<0.001$ \\
\hline
\end{tabular}

${ }^{*}$ p-value using t-tests, $\chi^{2}$ tests, or Fisher's exact tests as appropriate. PSA: Post-Stroke Anxiety

Table 2. Cross-sectional associations between functional outcomes and post-stroke anxiety (PSA) status

\begin{tabular}{|c|c|c|c|c|c|c|c|c|c|c|}
\hline & \multicolumn{5}{|c|}{ At 2 weeks } & \multicolumn{5}{|c|}{ At 1 year } \\
\hline & \multirow{2}{*}{$\begin{array}{l}\text { No PSA } \\
(\mathrm{N}=396)\end{array}$} & \multirow{2}{*}{$\begin{array}{c}\text { PSA } \\
(\mathrm{N}=27)\end{array}$} & \multicolumn{3}{|c|}{ Point difference } & \multirow{2}{*}{$\begin{array}{l}\text { No PSA } \\
(\mathrm{N}=276)\end{array}$} & \multirow{2}{*}{$\begin{array}{c}\text { PSA } \\
(\mathrm{N}=30)\end{array}$} & \multicolumn{3}{|c|}{ Point difference } \\
\hline & & & $\mathrm{t}$ & p-value* & p-value ${ }^{\dagger}$ & & & $\mathrm{t}$ & p-value* & p-value ${ }^{\dagger}$ \\
\hline $\begin{array}{l}\text { National Institutes of } \\
\text { Health Stroke Scale, } \\
\text { mean (SD) }\end{array}$ & $2.1(2.2)$ & $2.6(3.2)$ & -0.835 & 0.411 & 0.253 & $1.0(1.6)$ & $2.3(2.5)$ & -3.816 & $<0.001$ & $<0.001$ \\
\hline $\begin{array}{l}\text { Barthel Index, } \\
\text { mean (SD) }\end{array}$ & $86.0(18.7)$ & $81.3(20.9)$ & 1.136 & 0.265 & 0.201 & $94.3(12.3)$ & $78.5(23.8)$ & 5.953 & $<0.001$ & $<0.001$ \\
\hline $\begin{array}{l}\text { Mini-Mental State } \\
\text { Examination, mean(SD) }\end{array}$ & $24.7(4.1)$ & $22.8(4.7)$ & 2.242 & 0.025 & 0.093 & $25.7(4.2)$ & $22.0(5.3)$ & 4.062 & $<0.001$ & $<0.001$ \\
\hline
\end{tabular}

\section{Longitudinal associations between PSA at acute phase and changes in functional outcomes}

The longitudinal associations between PSA during the acute phase and changes in the three functional outcomes over 1 year in the 306 participants who completed the followup evaluation are displayed in Figure 2. No significant group differences were observed for any of the three outcomes. However, there were significant group $\times$ time interactions for changes in the NIHSS and BI score after adjusting for gender, history of depression, and baseline HAMD score. The NIHSS and BI scores worsened over 1 year in the presence of PSA at baseline. 


\section{DISCUSSION}

The main findings of this longitudinal study in a stroke cohort were that PSA at the acute phase was not associated with functional outcomes at the cross-sectional point, but predicted a worsening of stroke severity outcome and ADLs 1 year after stroke. PSA during the chronic phase was cross-sectionally associated with poor functional outcome 1 year after stroke. All associations were independent of potential covariates.

PSA was not associated with the functional status of stroke at the baseline examination. This result was discordant with that from PSD, which has been reported to be significantly associated with poor functional status during the acute phase of stroke. ${ }^{5,22}$ However, it was consistent with previous studies on the associations between PSA and functional outcomes during the acute phase. ${ }^{8}$ Stroke is a life-threatening attack that results in severe stress to patients. ${ }^{23}$ Therefore, anxiety may develop due to the stroke itself rather than to stroke functional status. A novel finding of this study was that PSA during the acute phase predicted a worsening of long-term stroke severity and ADLs over the 1-year time span, i.e., patients with PSA during the acute phase showed a worsening trajectory in neurological severity and physical disability, whereas those without PSA showed a generally improved disease prognosis (Figure 2). Although the finding on cognitive function measured with the MMSE was not significant (group $\times$ time interaction $\mathrm{p}=0.099$ ), it also showed a similar trend. Several explanations are plausible. First, treatment after a stroke becomes focused on improving general health and functional status. The presence of anxiety at this time can reduce social engagement, independence in ADLs, and health-related quality of life of survivors. ${ }^{8,24,25}$ Second, anxiety may lead to a pessimistic outcome of rehabilitation resulting in poor adherence to treatments. ${ }^{26}$ Third, comorbidity with depression should be considered, as PSD is a well-known predictor of poor stroke outcome..$^{27,28}$ In this study, depressive symptoms measured by the HAMD at baseline were significantly associated with PSA during the acute and chronic phases of stroke, consistent with a previous study. ${ }^{29}$ However, the association between PSA during the acute phase and worsening of functional status remained significant after adjusting for depressive symptoms.

Additionally, PSA was associated with a poor functional outcome during the chronic phase of stroke. This is a crosssectional finding that replicates previous studies with a similar design. ${ }^{5,7,8}$ However, this finding is particularly important when considering that PSA was not associated with any functional outcomes during the acute phase of stroke. Unlike the acute phase when patients are overwhelmed by a stroke, patients are more coherently aware of their functional status during the chronic phase; therefore, those with severe stroke
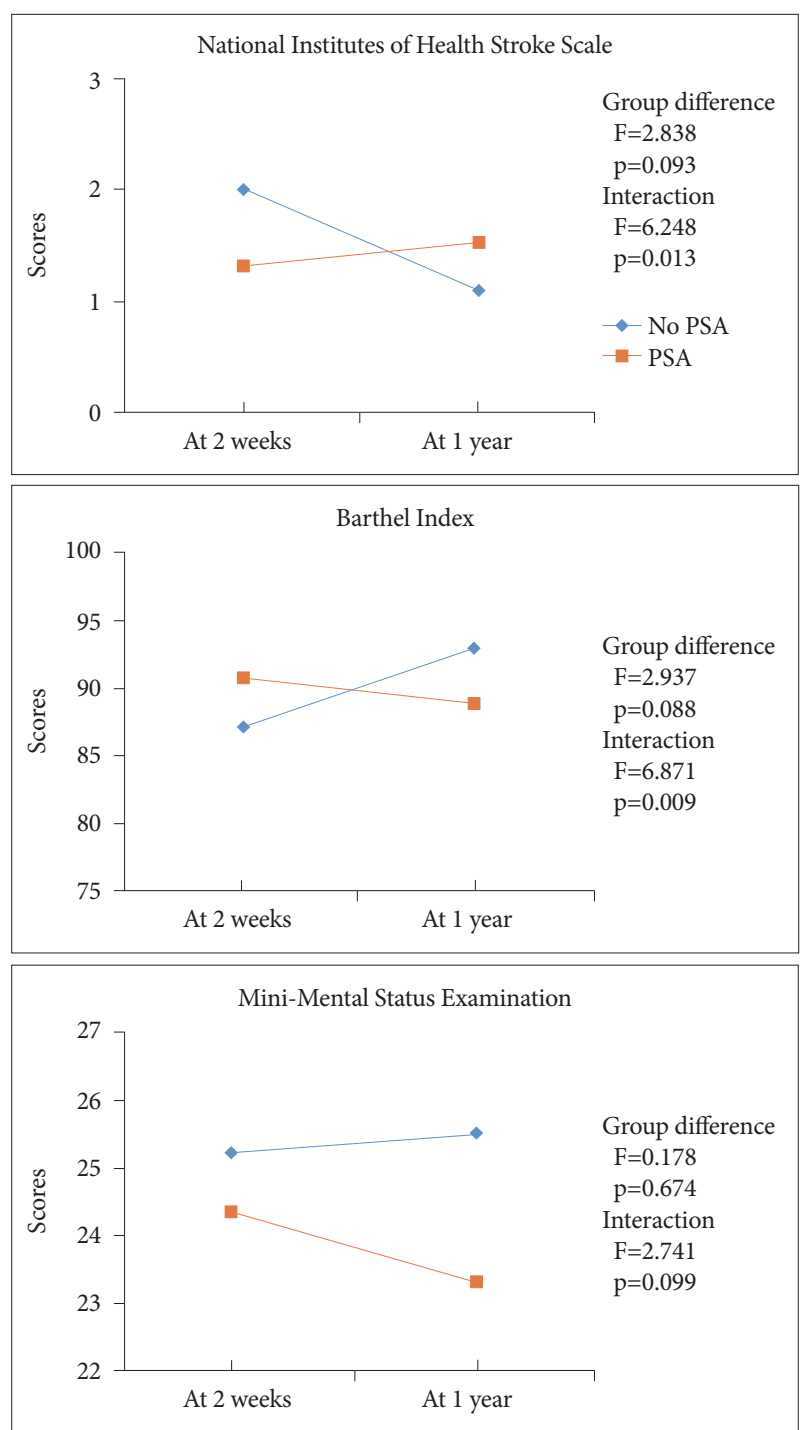

Figure 2. Longitudinal impact of Post-Stroke Anxiety (PSA) at 2 weeks on functional outcomes over 1 year after stroke.

are more vulnerable to anxiety. This finding suggests that PSA may be a state marker of functional outcome during the chronic but not the acute phase of stroke.

Before drawing a conclusion, several issues should be considered. First, the prevalence of PSA in this study was lower (6.4\% at 2 weeks and $9.8 \%$ at 1 year after stroke) compared to that in other studies, such as approximately $25 \%$ in meta-analyses. ${ }^{29,30}$ This can be explained by the strict recruitment criteria of the present study, as patients with severe cognitive impairment or aphasia who could not complete the self-rating scales or psychiatric interview were excluded from the study; thus, the generalizability of our results is limited to people with mild to moderate stroke severity without these deficits. Second, although the HADS is a well-validated and simple approach that has been used in previous studies on stroke, our study has limi- 
tation of using only a self-reported questionnaire HADS to assess PSA, not using a structured diagnostic interview. According to recent study, subtypes of anxiety disorder in stroke patients are important and phobic predominant patients are associated with poorer patient outcomes. ${ }^{31}$ Further research will also need to investigate the functional consequences of post stroke anxiety subtype based on structured diagnostic interview. Third, there was attrition in the recruitment process, although no difference was found between participants vs. nonparticipants in any of the characteristics evaluated at baseline.

The present study had several strengths. This study was the first to investigate the longitudinal associations between functional status and PSA by assessing the presence of anxiety and a range of covariates with well-validated assessment scales at similar time points ( 2 weeks and 1 year after stroke) in all participants. Because the etiology of anxiety may differ according to the time elapsed after stroke, the particular design used in the present study diminished the risk of errors due to different examination times. Additionally, the participants were consecutively recruited from among all eligible patients being treated for a recent stroke at the study hospital, which reduced the possibility of selection bias and increased potential generalizability.

In conclusion, screening for anxiety and depression is recommended even during the acute phase of stroke considering their independent detrimental effects on functional prognosis. Based on our findings, future studies should clarify the impact of acute phase PSA on long-term functional outcomes after stroke, such as the treatment effects of PSA using randomized controlled study designs and to establish the underlying mechanisms, including biological, behavioral, and social factors that contribute to this relationship, such as treatment adherence and trait negative response bias. In addition, screening for anxiety during the chronic phase is also encouraged, as this may reflect the functional status of stroke.

\section{Acknowledgments}

This study was supported by a grant of National Research Foundation of Korea Grant (NRF-2015M3C7A1028899), and was supported by a grant (BCRI 18018) Chonnam. National University Hospital Biomedical Research Institute to Prof. JM Kim.

\section{Conflicts of Interest}

The authors have no potential conflicts of interest to disclose.

\section{Author Contributions}

Conceptualization: Jae-Min Kim. Data curation: Hee-Ju Kang, Jae-Min Kim. Formal analysis: Eun-Hye Lee, Ju-Wan Kim, Hee-Ju Kang, SungWan Kim, Jae-Min Kim. Funding acquisition: Jae-Min Kim. Investigation: Eun-Hye Lee, Ju-Wan Kim, Hee-Ju Kang. Methodology: Sung-Wan Kim, Jae-Min Kim. Project administration: Jae-Min Kim, Ki-Hyun Cho. Resources: Man-Seok Park, Joon-Tae Kim, Ki-Hyun Cho. Software: Eun-Hye Lee, Ju-Wan Kim, Hee-Ju Kang. Supervision: Sung-Wan Kim, Jae-Min Kim. Validation: Sung-Wan Kim, Jae-Min Kim. Visualization: Eun-Hye
Lee, Jae-Min Kim. Writing-original draft: Eun-Hye Lee, Jae-Min Kim. Writing_review \& editing: Ju-Wan Kim, Hee-Ju Kang, Sung-Wan Kim, Jae-Min Kim.

\section{ORCID iDs}

Jae-Min Kim

Eun-Hye Lee

https://orcid.org/0000-0001-7409-6306

https://orcid.org/0000-0002-2597-9978

\section{REFERENCES}

1. Kutlubaev MA, Hackett ML. Part II: predictors of depression after stroke and impact of depression on stroke outcome: an updated systematic review of observational studies. Int J Stroke 2014;9:1026-1036.

2. Bartoli F, Lillia N, Lax A, Crocamo C, Mantero V, Carrà G, et al. Depression after stroke and risk of mortality: a systematic review and meta-analysis. Stroke Res Treat 2013;2013:862978.

3. Barker-Collo SL. Depression and anxiety 3 months post stroke: prevalence and correlates. Arch Cli Neuropsychol 2007;22:519-531.

4. Castillo CS, Schultz SK, Robinson RG. Clinical correlates of early-onset and late-onset poststroke generalized anxiety. Am J Psychiatry 1995;152:1174-1179.

5. Schultz SK, Castillo CS, Kosier JT, Robinson RG. Generalized anxiety and depression: assessment over 2 years after stroke. Am J Geriatr Psychiatry 1997;5:229-237.

6. Lincoln NB, Brinkmann N, Cunningham S, Dejaeger E, De Weerdt W, Jenni W, et al. Anxiety and depression after stroke: a 5 year follow-up. Disabil Rehabil 2013;35:140-145.

7. Ayerbe L, Ayis SA, Crichton S, Wolfe CD, Rudd AG. Natural history, predictors and associated outcomes of anxiety up to 10 years after stroke: the South London Stroke Register. Age Ageing 2014;43:542-547.

8. Li W, Xiao WM, Chen YK, Qu JF, Liu YL, Fang XW, et al. Anxiety in patients with acute ischemic stroke: risk factors and effects on functional status. Front Psychiatry 2019;10:257.

9. Maaijwee NA, Tendolkar I, Rutten-Jacobs LC, Arntz RM, Schaapsmeerders P, Dorresteijn LD, et al. Long-term depressive symptoms and anxiety after transient ischaemic attack or ischaemic stroke in young adults. Eur J Neurol 2016;23:1262-1268.

10. Zigmond AS, Snaith RP. The hospital anxiety and depression scale. Acta Psychiatr Scand 1983;67:361-370.

11. Aben I, Verhey F, Lousberg R, Lodder J, Honig A. Validity of the beck depression inventory, hospital anxiety and depression scale, SCL-90, and hamilton depression rating scale as screening instruments for depression in stroke patients. Psychosomatics 2002;43:386-393.

12. Brennan C, Worrall-Davies A, McMillan D, Gilbody S, House A. The Hospital Anxiety and Depression Scale: a diagnostic meta-analysis of case-finding ability. J Psychosom Res 2010;69:371-378.

13. Oh SM, Min KJ, Park DB. A study on the standardization of the hospital anxiety and depression scale for Koreans: a comparison of normal, depressed and anxious groups. J Korean Neuropsychiatr Assoc 1999; 38:289-296.

14. Kasner SE, Chalela JA, Luciano JM, Cucchiara BL, Raps EC, McGarvey ML, et al. Reliability and validity of estimating the NIH stroke scale score from medical records. Stroke 1999;30:1534-1537.

15. Mahoney FI, Barthel DW. Functional evaluation: The Barthel Index. Md State Med J 1965;14:61-65.

16. Kang Y, Na DL, Hahn S. A validity study on the Korean Mini-Mental State Examination (K-MMSE) in dementia patients. J Korean Neurol Assoc 1997;15:300-308.

17. Menlove L, Crayton E, Kneebone I, Allen-Crooks R, Otto E, Harder H. Predictors of anxiety after stroke: a systematic review of observational studies. J Stroke Cerebrovasc Dis 2015;24:1107-1117

18. Kootker JA, van Mierlo ML, Hendriks JC, Sparidans J, Rasquin SM, de Kort PL, et al. Risk factors for symptoms of depression and anxiety one year poststroke: a longitudinal study. Arch Phys Med Rehabil 2016;97:919-928. 
19. Wright F, Wu S, Chun HY, Mead G. Factors associated with poststroke anxiety: a systematic review and meta-analysis. Stroke Res Treat 2017;2017:2124743.

20. Sheehan DV, Lecrubier Y, Sheehan KH, Amorim P, Janavs J, Weiller E, et al. The mini-international neuropsychiatric interview (M.I.N.I): the development and validation of a structured diagnostic psychiatric interview for DSM-VI and ICD-10. J Clin Psychiatry 1998;59:22-33.

21. Hamilton M. A rating scale for depression. J Neurol Neurosurg Psychiatry 1960;23:56-62.

22. Kang HJ, Bae KY, Kim SW, Lee EH, Kim JT, Park MS, et al. Impact of acute phase depression on functional outcomes in stroke patients over 1 year. Psychiatry Res 2018;267:228-231.

23. Mukherjee D, Levin RL, Heller W. The cognitive, emotional, and social sequelae of stroke: psychological and ethical concerns in post-stroke adaptation. Top Stroke Rehabil 2006;13:26-35.

24. Astrom M. Generalized anxiety disorder in stroke patients. A 3-year longitudinal study. Stroke 1996;27:270-275.

25. Shimoda K, Robinson RG. Effects of anxiety disorder on impairment and recovery from stroke. J Neuropsychiatry Clin Neurosci 1998; 10:34-40.

26. Masskulpan P, Riewthong K, Dajpratham P, Kuptniratsaikul V. Anxiety and depressive symptoms after stroke in 9 rehabilitation centers. J Med Assoc Thai 2008;91:1595-1602.

27. Cai W, Mueller C, Li YJ, Shen WD, Stewart R. Post stroke depression and risk of stroke recurrence and mortality: a systematic review and meta-analysis. Ageing Res Rev 2019;50:102-109.

28. Robinson RG, Jorge RE. Post-stroke depression: a review. Am J Psychiatry 2016;173:221-231.

29. Campbell Burton CA, Murray J, Holmes J, Astin F, Greenwood D, Knapp P. Frequency of anxiety after stroke: a systematic review and meta-analysis of observational studies. Int J Stroke 2013;8:545-559.

30. Hackett ML, Yapa C, Parag V, Anderson CS. Frequency of depression after stroke: a systematic review of observational studies. Stroke 2005; 36:1330-1340.

31. Chun HY, Whiteley WN, Dennis MS, Mead GE, Carson AJ. Anxiety after stroke: the importance of subtyping. Stroke 2018;49:556-564. 